\title{
THE HIGH CURRENT TRANSPORT EXPERIMENT FOR HEAVY ION INERTIAL FUSION*
}

\author{
P.A. Seidl, D. Baca, F. M. Bieniosek, C.M. Celata, A. Faltens, L. R. Prost, G. Sabbi, W. L. Waldron, \\ Lawrence Berkeley National Laboratory, Berkeley, CA 94720 USA \\ R. Cohen, A. Friedman, S.M. Lund, A.W. Molvik \\ Lawrence Livermore National Laboratory, Livermore, CA 94550 USA \\ I. Haber \\ University of Maryland, College Park, MD 20742 USA
}

\begin{abstract}
The High Current Experiment (HCX) at Lawrence Berkeley National Laboratory is part of the US program to explore heavy-ion beam transport at a scale representative of the low-energy end of an induction linac driver for fusion energy production. The primary mission of this experiment is to investigate aperture fill factors acceptable for the transport of space-charge-dominated heavy-ion beams at high space-charge intensity (linecharge density $\sim 0.2 \square \mathrm{C} / \mathrm{m}$ ) over long pulse durations ( $>4$ $\square \mathrm{s}$ ) in alternating gradient electrostatic and magnetic quadrupoles. This experiment is testing -- at driverrelevant scale -- transport issues resulting from nonlinear space-charge effects and collective modes, beam centroid alignment and beam steering, matching, image charges, halo, electron cloud effects, and longitudinal bunch control. We present the results for a coasting $1 \mathrm{MeV} \mathrm{K}^{+}$ ion beam transported through the first ten electrostatic transport quadrupoles, measured with beam-imaging and phase-space diagnostics. The latest additions to the experiment include measurements of the secondary ion, electron and atom coefficients due to halo ions scraping the wall, and four magnetic quadrupoles to explore similar issues in magnetic channels.
\end{abstract}

\section{INTRODUCTION}

The High Current Experiment (HCX) [1] located at Lawrence Berkeley National Lab and carried out by the HIF-VNL (Heavy-Ion Fusion Virtual National Laboratory: a collaboration between groups at LBNL, LLNL and Princeton Plasma Physics Laboratory, which has the goal of developing heavy-ion accelerators capable of igniting inertial-fusion targets for electric power production) is designed to explore the physics of intense beams with line-charge density of about $0.2 \square \mathrm{C} / \mathrm{m}$ and pulse duration $4<\square<10 \square \mathrm{s}$, close to the values of interest for a fusion driver. Experiments are performed near driver injection energy (1-1.8 MeV). HCX beam transport is at present mainly based on electrostatic quadrupole focusing, which provides efficient transport at low energy and provides clearing fields which sweep out unwanted electrons. However, magnetic transport experiments have commenced, to gain operational experience and to explore

* Supported by the Office of Energy Research, US DOE, at LBNL \& LLNL, contract numbers DE-AC03-76SF00098 and W-7405-Eng-48. special limitations associated with magnetic focusing, in particular the onset of transport-limiting effects due to electrons trapped in the potential well of the ion beam.

\section{EXPERIMENTAL CONFIGURATION}

The present configuration begins with the $\mathrm{K}^{+}$ion source and injector, an electrostatic quadrupole matching section (six quadrupoles), and the first 10 electrostatic transport quadrupoles.

A multi-purpose diagnostic station (D-end) is at the end of the beam line (Fig. 1). Beam diagnostics are also located at the interface of the matching section and the ten transport quadrupoles (QD1) and after the last (D2) transport quadrupole in the periodic lattice. They include transverse slit scanners, Faraday cups and current transformers. The Gas and Electron Source Diagnostic (GESD) is located at the end of D-end.

Most measurements so far have been made at $1.0 \mathrm{MeV}$, to avoid any high voltage insulation issues in the injector. Though much of the beam physics should scale predictably between 1 and $2 \mathrm{MeV}$, future measurements will verify this, and also establish operating experience at higher injection energy. The beam energy at present is limited to $1.5 \mathrm{MeV}$ until the water resistor that distributes the voltage along the injector column is modified. The column has operated at 1.8 MV during checks of the injector optics modifications.

To date, contact-ionization and alumino-silicate ion sources have been used. The injector beam characterization measurements and the first measurements through the HCX were made using the contact ionization source, before switching to the alumino-silicate source (100 mm diameter) in April 2002. Earlier versions of alumino-silicate sources suffered from poor currentdensity uniformity. There was a considerable aluminosilicate large-source R\&D effort during 2001-2 aimed at improving the uniformity. As a consequence of improvements to the diode optics and the improvements to the ion source [2], the hollowness of the beam has decreased from $20 \%$ to $10 \%$. The emittance has decreased from $\square_{h}=10 \times 10^{-7}$ to $6 \times 10^{-7} \mathrm{~m}$ compared to the theoretical minimum, $\square_{n}=2 R \sqrt{k T / m}=1.7 \square 10^{\square 7} \mathrm{~m}$ where $\mathrm{T}$ is the source temperature and $\mathrm{R}$ is the radius. The experimental current is within $2 \%$ of the expected value based on 3D PIC simulations. 


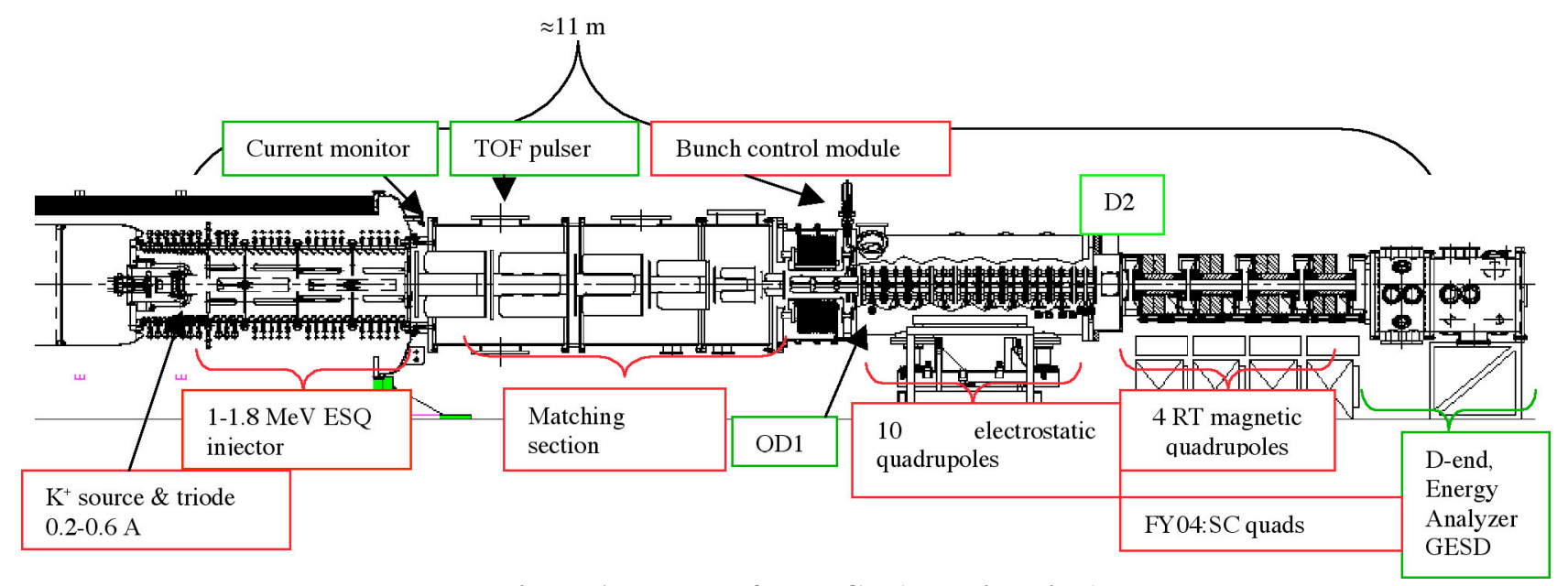

Figure 1: Layout of the HCX (elevation view).

In the operating range from 1.0 to $1.8 \mathrm{MeV}$ the beam brightness $\left(\mathrm{B}_{\mathrm{n}}=\mathrm{I} / \square_{\mathrm{n}}^{2}\right)$ is $0.7<\mathrm{B}_{\mathrm{n}}<2 \mathrm{~A} / \mathrm{mm}^{2}$, and the number of ions per pulse is $6 \times 10^{12}<\mathrm{N}<1.7 \times 10^{13}$.

First beam was transported in the HCX in January 2002, less that one month after the assembly of the initial configuration.

\section{MATCHING AND TRANSPORT THROUGH ELECTROSTATIC QUADRUPOLES}

The six-quadrupole matching section is designed to compress the beam area transversely by a factor $\approx 25$ and produce the matched beam parameters for periodic transport in the electrostatic lattice. In this significant beam manipulation the radii of the first (QM1) and last (QM6) matching quadrupole bores are $r_{p}=100 \mathrm{~mm}$ and 31 $\mathrm{mm}$ respectively, and the maximum envelope excursions occur in the first and second quadrupoles, with the beam filling radii up to $0.8 \cdot \mathrm{r}_{\mathrm{p}}$. The beam centroid exiting the injector is offset from the beam line axis by a few millimeters and milliradians, and the centroid undergoes betatron oscillations through the first three (QM1-3) quadrupoles of the matching section until being corrected in QM4-6. QM4-6 may each be displaced in the horizontal and vertical directions by $\pm 15 \mathrm{~mm}$ to correct the beam centroid offset. Matching (QM1-6) and transport quadrupole (Q1-10) voltages range up to $\pm 43 \mathrm{kV}$, and focusing gradients up to $10 \mathrm{kV} / \mathrm{cm}^{2}$. (Experiments at higher injection energy will require proportional increase in the quadrupole potentials to achieve a nearly identical envelope solution.)

Although the beam fills a relatively large fraction of the aperture in the early part of the matching section, pickup signals capacitively coupled to the quadrupole electrodes indicate that beam loss is less than $0.5 \%$ through the middle, or "flattop" of the beam pulse. Beam loss at the head and tail of the current pulse may be greater, and is presently being studied. The pickup signal due to lost ions is effectively amplified by the large secondary electron coefficient [3], making this diagnostic more sensitive to beam loss than comparisons of the total beam current data at the injector exit to that at QD1. The Faraday cupcurrent transformer ratio is $>95( \pm 2) \%$.
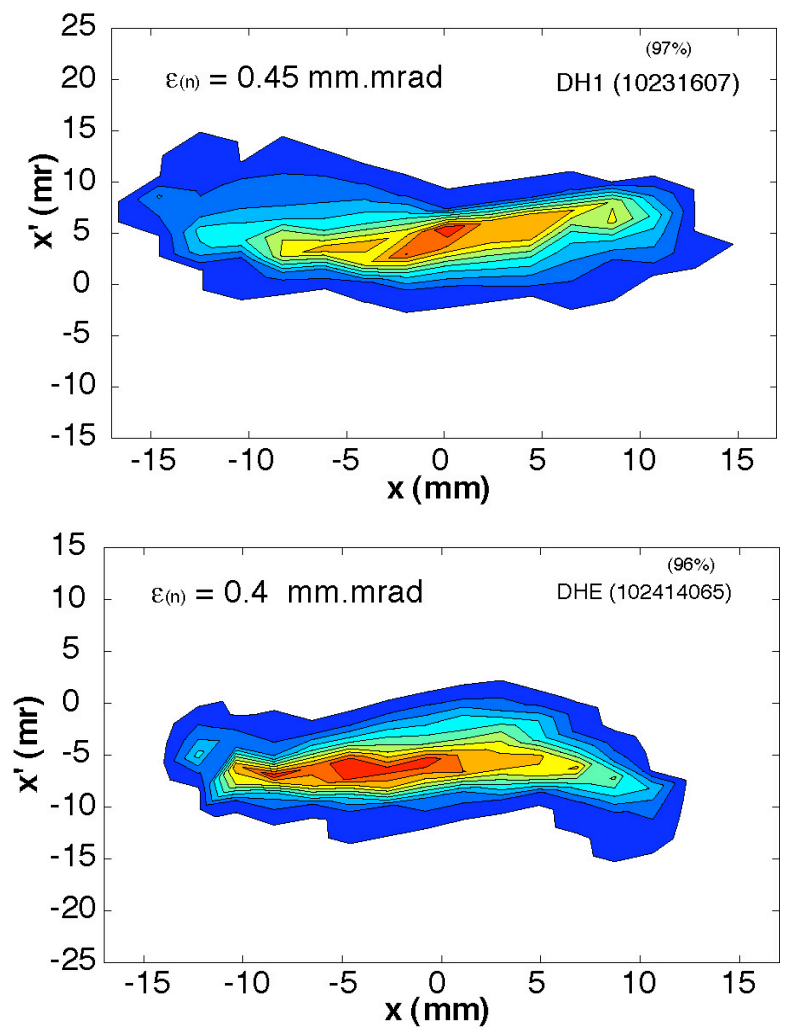

Figure 2: Horizontal phase space (a) before and (b) after the 10 quadrupole periodic transport lattice, for the $80 \%$ fill factor case, for a time slice near mid-pulse.

The steering quadrupole displacements $( \pm 1-4 \mathrm{~mm}$ typical) are determined by calculating the (single particle) trajectory through QM4-6 for a misaligned beam, and then solving for the required displacements. Three steering quadrupoles QM4-6) are used to align the beam centroid at QD1 (instead of the minimum of two displacements) subject to the additional constraint of minimizing the sum of displacements of the lenses. Following such a procedure, the beam centroid positions 
$(<\mathrm{x}>,<\mathrm{y}>)$ and angles $\left(<\mathrm{x}^{\prime}>,<\mathrm{y}^{\prime}>\right)$ are routinely within $0.5 \mathrm{~mm}$ and $2 \mathrm{mrad}$ of the central axis of the channel.

The horizontal phase space at QD1 is shown in Fig. 2a. The stability and reproducibility of the envelope measurements have been characterized by a standard deviation of $\approx 0.3 \mathrm{~mm}$ and $\approx 1 \mathrm{mrad}$ among five repeated measurements. The achievable beam envelope and centroid control are key ingredients in determining the allowable filling factor as determined by beam loss and emittance growth. Integrating the envelope equation through the ten-quadrupole lattice to D2 (initialized with QD1 measures of envelope radii, convergence angles, current, and D-end measurements of beam energy) gives a calculated envelope in agreement with the experiment at the D2 location to within $0.4 \mathrm{~mm}$ and $3 \mathrm{mrad},(<10 \%)$. Early calculations of the envelope showed a larger discrepancy, which led to an analysis of several effects, which resolved most of the disagreement. The effects are: (1) Realistic fringe field model based on 3D field calculations; (2) quadrupole $\mathrm{E}_{\mathrm{z}}$ and corresponding radial focusing force; (3) corrections for the grounded slit plates of the intercepting diagnostics that short out the self-field of the beam near the diagnostic; and (4) more accurate determination of the beam current and energy.

We have made two fill-factor measurements, for the envelope filling $\approx 60 \%$ and $80 \%$ of the available bore diameter in the transport channel. Each case requires a different matching solution in the matching section, and the fill factor (ratio of the beam major radius to aperture radius) is increased by tuning the upstream beam to the matched beam conditions in the transport section for a lower focusing gradient, rather than by changing the current.

PIC simulations [4] predict that matched beam excursions filling $80 \%$ of the quadrupole bore would result in negligible emittance growth, if perfect alignment and envelope control were maintained. Within the experimental sensitivity there is no evidence of emittance growth at the end of the electrostatic lattice for both $60 \%$ and $80 \%$ fill factor cases. Systematic biases such as those due to finite slit widths and rotational misalignments are still being analyzed, and may contribute an uncertainty of $10-20 \%$ to the emittance. Figure 2(b) show the horizontal beam phase space in the converging plane for the more aggressive $80 \%$-filling factor of the $46 \mathrm{~mm}$-diameter aperture. Since the transportable current scales as the square of the fill factor, determining its maximum will have a large impact on the cost of multi-beam induction accelerators for HIF.

In the entire distance, and for both fill factor cases, currents collected on quadrupole electrodes indicate particle losses of $<0.5 \%$, while Faraday cup current monitors indicate $\approx 1 \%$ losses. Halo measurements indicate that the beam profile intensity $\approx 5 \mathrm{~mm}$ from the hard-edge of the beam distribution falls to the order of $10^{-4}$ of the peak density in the core of the beam, the sensitivity limit with present slit-cup diagnostics.

In the context of multiple beam arrays for heavy-ion fusion, electrostatic quadrupoles composed of cylindrical electrodes make a compact unit cell. For the beam measurements to date, the current density averaged over a unit cell is $\langle\mathrm{J}\rangle \approx 40 \mathrm{~A} / \mathrm{m}^{2}$ (denominator includes the area occupied by the beam focusing electrodes between adjacent channels of an array), and future measurements at $1.8 \mathrm{MeV}$ will increase this by $2-3 \mathrm{x}$. Furthermore, it appears from these measurements that higher $\langle\mathrm{J}\rangle$ might be possible. Compiling data to determine the optimum filling factor is a principal goal of the experiment.

\section{BEAM CHARGE DISTRIBUTION}

We observe a diamond-shaped beam pattern at QD1 (Fig. 3) and at D-end, which is attributed to nonlinear fields (that is, anharmonic field components not varying in direct proportion to the transverse coordinates) in the ESQ injector and electrostatic image forces in the matching and transport section. All the $J(x, y)$ data will be used with the phase space data at QD1 to construct a consistent particle distribution for simulation studies [5].

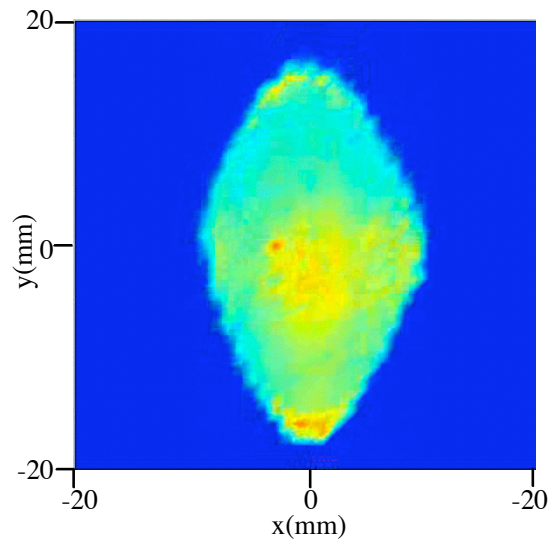

Figure 3: Beam current density profile $\mathrm{J}(\mathrm{x}, \mathrm{y}$, $\mathrm{t} \approx$ midpulse) measured with crossed slits at QD1.

The time-resolved crossed-slit data show that at QD1 the profile of the beam during the rise and fall of the beam current pulse is larger than during the flattop. Ballooning of the beam head was predicted for the beam exiting the injector from 3D particle-in-cell simulations and is attributed to a voltage risetime in the diode which is presently too slow to match the space-charge evolution of the current. Calculations of the head-tail dynamics through the rest of the HCX are underway.

\section{TRANSVERSE PHASE SPACE DIAGNOSTIC DEVELOPMENT}

In prototype tests, we have confirmed the utility of optical diagnostics for rapid and highly detailed measurements of beam phase space distributions [6].

Figure 4 shows an image from a typical slit scan from a prototype optical emittance scanner. The beam is imaged through the existing slits, already utilized in the conventional emittance scanner (Fig. 2), onto a thin alumina ceramic wafer, where charge buildup is prevented from secondary electron emission by a grounded wire grid placed directly on the screen. The response time of the 
alumina is shorter than the rise (200 ns) and fall time of the beam current pulse. The images are viewed behind the wafer with a gated, image-intensified CCD camera. The slit is oriented vertically and is moved horizontally by a step motor. The images can be integrated to provide a simulated slit scan for comparison with conventional slit scanner data. A large amount of new phase space correlation information is available from the shapes of the images. Detailed analysis of the data is underway [5]. The technique will be used in the more crowded diagnostic gap at D1, and slits will be replaced with a pepper-pot hole arrangement, which will provide fullycorrelated four-dimensional transverse phase space data.
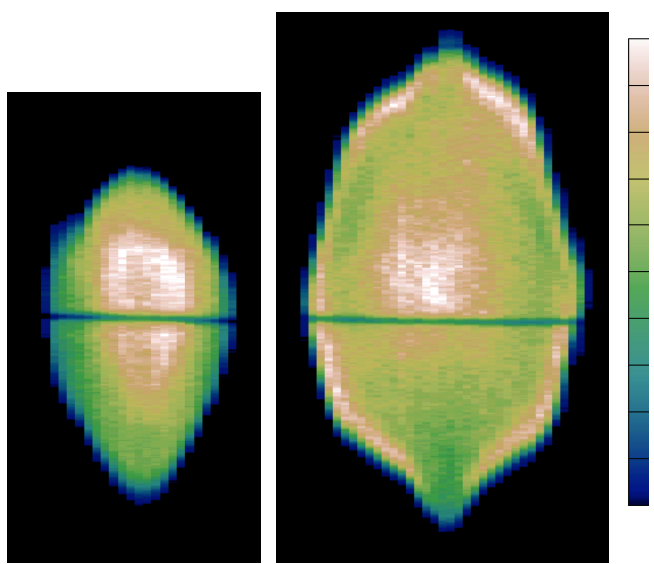

Figure 4: Beam intensity profile for $60 \%$ (left) and $80 \%$ (right) fill factor at the D2 location based on measured optical slit scan, referred to the horizontal slit plane. The scale for the images are $2.28 \times 4.26 \mathrm{~cm}$ and $3.11 \times 5.15 \mathrm{~cm}$.

\section{MEASUREMENT OF SECONDARY ELECTRONS AND ATOMS}

The Gas-Electron Source Diagnostic (GESD) is designed to measure the gas desorption and secondary electron and ion emission due to heavy-ion beams impacting a surface [3]. Approximately $0.1 \%$ of the beam current passes through a small aperture and strikes a target, adjustable between angles of incidence from $75^{\circ}$ to $88^{\circ}$ relative to normal.

We observed a significant variation in secondary electron emission with the angle of the target (yield varies from $30-130$ between $78^{\circ}$ and $88^{\circ}$ ). Beam ions lost to the walls will be near grazing incidence, and therefore will have the higher values of secondary emission coefficient.

We find the gas desorption yield varies from $8-12 \mathrm{x}$ $10^{3} /$ ion, with the angular dependence and magnitude similar to CERN data [7] with different beam parameters.

A beam line of four pulsed magnetic quadrupoles, instrumented with diagnostics to measure the production and energy of trapped electrons, secondary atoms and ions was installed $[3,8]$ downstream of the first group of 10 electrostatic quads in April 2003, and will be a principal area of activity in the next year.

\section{DIAGNOSTICS FOR ABSOLUTE BEAM ENERGY MEASUREMENTS}

Two new diagnostics, an electrostatic energy analyzer (EA) and a time-of-flight pulser (TOF) were installed to more precisely determine the beam energy and to make longitudinal phase-space measurements.

The EA, a $90^{\circ}$-spectrometer with a radius of $46 \mathrm{~cm}$, and a gap of $2.5 \mathrm{~cm}$ was operated up to $\square \mathrm{V}=110 \mathrm{kV}$. The relative accuracy is $\pm 0.2 \%$, allowing us to follow variations in the beam energy as a function of time during the beam pulse. The calibration depends on the geometry and fringe fields of the analyzer. By changing the beam energy by a known absolute amount, we were able to provide an independent calibration: The beam passed through a $28 \%$-transparent hole-plate, and the gas cloud created at the hole-plate stripped singly charged $\mathrm{K}^{+}$beam ions to doubly charged $\mathrm{K}^{2+}$. The absolute calibration was determined by varying the electric potential at the plate, and thus the energy of the $\mathrm{K}^{2+}$ ions entering the EA.

For the TOF measurements, a fast pulser in the matching section ( $0.3 \square \mathrm{s}$ FWHM) induced $1 \%$ energy perturbations. These energy pulses manifest as 5-10\% current perturbations measured $5.4 \mathrm{~m}$ downstream. Figure 5 shows the perturbation on the beam current waveform measured downstream along with a theoretical calculation of the expected perturbation based on a 1-D cold-fluid model. Comparing the measured and expected delay time of the perturbation determines the absolute energy of the beam.

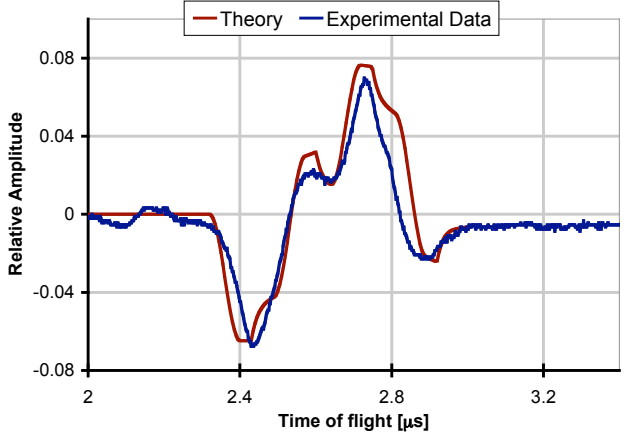

Figure 5: Cold fluid model vs. perturbation on the beam current waveform. Initial perturbation applied @ $\mathrm{t}=0$.

Both the TOF and EA diagnostics determine the absolute beam energy to $\pm 2 \%$, with both measurements agreeing within these uncertainties. The precise determination of the energy is essential for the agreement between envelope simulations and experimental data.

Fig. 6 shows the longitudinal energy distribution obtained with the EA. The 10\%-higher energy head and low-energy tail are understood to be from the beam longitudinal space charge, which accelerates particles at the front end of the bunch and decelerates particles at the rear of the pulse. These data also shows that in the middle of the pulse, the mean beam energy is constant to within $0.5 \%$ for $3.1 \square \mathrm{s}$. 


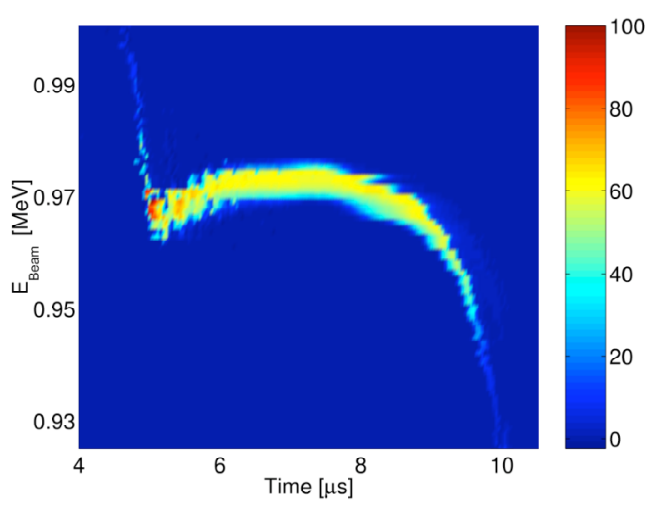

Figure 6: Longitudinal Energy Distribution measured with the electrostatic energy analyzer. $\mathrm{K}^{+}$ions were detected at the beam head with $1.1 \mathrm{MeV}$ (not visible on this scale).

This information (particularly the head and tail energy variations with respect to the core of the beam pulse) will be used to help complete the design of a bunch end control module [10] to be installed next year between the matching section and the periodic transport lattice. This represents a first step towards conducting more longitudinal physics experiments.

\section{SUPERCONDUCTING QUADRUPOLE DEVELOPMENT}

Superconducting magnets will be installed in HCX. While pulsed magnets provide a more flexible alternative for initial experiments, superconducting technology is the most attractive in view of the ultimate fusion driver application. Superconducting magnets based on flat coils have received considerable attention in recent years, due to their simplicity and cost-effectiveness. The HCX design uses double-pancake coils wound around iron cores, and was derived from an earlier design of a multiple beam quadrupole array for heavy ion fusion.

A prototype cryostat housing two quadrupole magnets (an FD doublet) is completed. The unit includes a shielded straight chimney, needed to maximize the space available for induction acceleration cores surrounding the transport line of an induction linac.

The measurement of the field quality of an optimized prototype quadrupole is commencing [9].

\section{CONCLUSIONS}

The transport results through the first ten electrostatic quadrupoles show good beam control, agreement with an improved envelope model, and indicate that transport at $80 \%$ beam filling factors at the front end of a heavy-ion induction linac might be possible with acceptable emittance growth and beam loss. Other fill factors will be measured. Details of the measured phase space distribution are being used to initialize particle-in-cell simulations for comparison of data with theoretical models. Prototype diagnostic tests show that higher data acquisition rates and sensitivity to more correlations between the transverse phase space measurements will enhance future experimental output.

Future measurements of secondary electrons, ions and atoms in magnetic quadrupoles will also have a direct impact on future heavy ion induction accelerators.

Because the injected beam is non-uniform, and inevitably mismatched to a small extent, the first few quadrupoles in the HCX lattice as well as the quads in the matching section provide a region where the beam distribution can relax. While 10 or 20 quads are too few for settling questions of emittance evolution in a long transport system, they are of the correct length for the rapid evolution of the emittance and beam profile, which is expected in the front end of an accelerator. An additional 20-30 quadrupoles would increase the transport length to $4.5-6 \cdot\left(2 \square \mathrm{v} / \square_{\mathrm{p}}\right)$ plasma periods. Theoretical models predict that this would be just enough to observe the relaxation of phase space and distribution inhomogeneities $[4,5]$. This leads to setting the bore diameter and choosing the optimum current.

\section{ACKNOWLEDGEMENTS}

We are grateful to R. Hipple, W. Strelo and their staff for excellent technical support.

\section{REFERENCES}

[1] P.A. Seidl, et al. Overview of the Scientific "Objectives of the High Current Experiment for Heavy-Ion Fusion" Proc. 2001 Part. Accel. Conf. pp. 2932-2934, IEEE \#01CH37268C. Piscataway, NJ 08855. S.S. Yu et al., "An updated point design for heavy ion fusion", to be published in Fusion Sci. Tech.

[2] J.W. Kwan et al, LASER AND PARTICLE BEAMS 20 (4), 2002. LBNL-50221.

[3] A.W. Molvik et al., "Initial experimental studies of electron accumulation in a heavy-ion beam", these proceedings.

[4] C.M. Celata et al., LASER AND PARTICLE BEAMS 20 (4) p.577 (2002). C.M. Celata et al., "Particle-in-cell simulations of the high current experiment", these proceedings.

[5] A.Friedman et al., "Simulation using initial 4D beam particle distributions synthesized from experimental data", these proceedings.

[6] F.M. Bieniosek et al., these proceedings.

[7] E. Mahner, et al., Phys. Rev. ST-AB, 013201, (2003).

[8] A.W. Molvik et al., LASER AND PARTICLE BEAMS 20 (4), 2002. LBNL-51009.

[9] G.L. Sabbi et al., these proceedings.

[10] C. Burkhart, "The Closed-loop Amplifier Regulated Driver, a High Accuracy, Low Cost Modulator for HIF Accelerators," US Dept. of Energy SBIR final Report, DE-FG03-00ER83010, May 2001. 